\title{
American Mineralogist thanks the 2019 reviewers
}

\begin{abstract}
American Mineralogist acknowledges the time and effort of the 2019 reviewers. The Journal thrives due to the commitment of many people. It could not do so without the support of crucial volunteers.
\end{abstract}

\section{REVIEWERS}

Achilles, C.N

Ackerson, M.

Adcock, C.T.

Adkins, J.

Agangi, A.

Airaghi, L.

Aja, S.

Alvaro, M.

Anenburg, M.

Angisboust, S.

Appold, M.

Arai, S.

Arato, R.

Arienzo, I.

Arzilli, F.

Ashley, K.T.

Austrheim, H.

Axler, J.A.

Ayers, J.C.

Backus, E.

Bagdassarov, N.

Balan, E.

Balcone-Boissard, H.

Baldermann, A.

Balić-Žunić, T.

Barboni, M.

Barnes, C.G.

Baronnet, A.

Basu, A.

Bebout, G.E.

Befus, K.S.

Bell, A.S.

Belmonte, D.

Belonoshko, A.

Belviso, C.

Bénard, A.

Bendaoud, A.

Benzerara, K.

Bergantz, G.

Berger, A.

Biagioni, C.

Bilenker, L.D.

Blereau, E.

Bloch, Elias
Boffa Ballaran, T.

Bolfan-Casanova, N.

Bonadiman, C.

Borda, M.

Bosi, F.

Böttcher, M.E.

Boyce, J.

Boyd, E.

Brey, G.P.

Brigatti, M.F.

Broadley, M.

Bromiley, G.D.

Brounce, $\mathrm{M}$.

Brown, M.

Brown, P.E.

Brownlee, S.J.

Bureau, H.

Burgess, K.

Burnham, A.D.

Burnley, P.C

Burns, P.C.

Calzaferri, G.

Camara, F.

Campbell, A.

Campbell, L.S.

Campomenosi, N.

Caricchi, L.

Carpenter, M.A.

Carrez, P.

Cempírek, J.

Cenki-Tok, B.

Centrella, S.

Cernok, A.

Chamberlain, K.

Chang, Y-Y.

Chappaz, A.

Chappell, C.

Chatterjee, N.

Chelle-Michou, C.

Chemtob, S.

Chen, B.

Chen, T.

Chen, W.

Cheng, $\mathrm{H}$.

Cheng, L.

Cherniak, D.J.

Chin, E.

Chou, I-M.

Christiansen, E.

Christidis, G.E.

Christy, A.G.
Chu, X.

Chukanov, N.V.

Churchman, G.

Cisneros, $\mathrm{M}$.

Clark, A.

Clark, C.

Colomban, P.

Cook, N.J.

Cormack, A.

Corrigan, C.

Cortes, J.

Cottrell, E.

Courtin-Nomade, A.

Cui, $\mathrm{H}$.

Dai, L.

Dalou, C.

Daniel, I.

Danyushevsky, L.

Das, $\mathrm{S}$.

Davis, F.A.

Davis, $P$.

Deditius, A.P.

Deering, C.

Del Real, I.

Della Ventura, G.

Deng, X.-D.

Dera, P.K.

Deschanels, X.

Di Renzo, F.

Dilles, J.H.

Dobrzhinetskaya, L.

Dohmen, R.

Dolejs, D.

Dong, $\mathrm{H}$.

Dorfman, S.M.

Dove, P.M.

Dragovic, B.

Duffy, T.S.

Duncan, $\mathrm{M}$.

Dutta, R.

Dyar, M.D.

Easton, R.M.

Ebel, D.S.

Ehrig, K.

Elliott, C.

Elmi, C.

Elzinga, E.

Epple, M.

Eramo, G.

Estrade, G.

Etschamnn, B. 
Fanara, S.

Fedortchouk, Y.

Fei, H.

Fei, Y.

Ferraris, G.

Ferrero, S.

Filiberto, J.

Finkelstein, G.

Fischer, M.

Fischer, R.X.

Flament, N.

Forray, F.L.

Forshaw, J.

Forster, M.

Fortin, M.-A.

Fougerouse, D.

Frank-Kamenetskaya, O.

Franz, G.

Frost, R.L.

$\mathrm{Fu}, \mathrm{S}$.

Furman, T.

Gaboreau, S.

Gaboury, D.

Gaillot, A.-C.

Gaillou, E.

Galuskin, E.V.

Galuskina, I.

Galvez, M.

Ganguly, J.

Gao, S.

Garber, J.

Gates, W.P.

Gatta, G.D.

Geballe, Z.

Geiger, C.A.

Gemmi, M.

Gleason, A.

Gleeson, M.

Gloter, A.

Godelitsas, A.C.

Goldman, N.

Gonnermann, H.

González-Jiménez, J.M.

Goodell, P.

Goodrich, C.

Gorzelak, P.

Gou, H.

Grassmann, O.

Greenberg, E.

Gregoire, M.

Groat, L.A.

Grosvenor, A.

Guggenheim, S.J.

Ha, Y.

Hacker, B.R.

Hagen-Peter, G.

Haggerty, S.E.

Halenius, U.
Hallis, L.

Hammerli, J.

Hanchar, J.

Hao, J.

Harley, S.

Heck, P.R.

Henderson, G.S.

Henry, D.J.

Hetherington, C.

Hickey-Vargas, R.

Hilairet, N.

Hogmalm, J.

Hoisch, T.

Holness, M.

Holycross, M.

Hong, W.

Honório de Faria, T.

Hopp, J.

Horton, F.

$\mathrm{Hu}, \mathrm{J}$.

$\mathrm{Hu}, \mathrm{S}$.

$\mathrm{Hu}, \mathrm{X}$.

$\mathrm{Hu}, \mathrm{Y}$.

Hughes, J.M.

Hui, $\mathrm{H}$.

Hummer, D.R.

Huraiová, M.

Huston, D.

Hutchinson, M.

Le Losq, C.

Imai, $\mathrm{H}$.

Ingrin, J.

Isaak, D.G.

Ishii, $\mathrm{T}$.

Jacques, L.

Janots, E.

Jiang, H.

Jiang, S.-Y.

Jiménez-Millán, J.

Jing, $Z$.

Johnson, S.

Johnston, C.T.

Jolliff, B.L.

Jonckheere, R.C.

Jones, R.H.

Jordan, G.

Jung, H.S.

Kagi, H.

Kalinichev, A.

Kaminsky, F.

Karki, B.B.

Kendrick, M.A.

Kesler, S.E.

Ketcham, R.A.

King, P.L.

Kirkland, C.

Kiseeva, E.S.

Klein, F.
Koch-Müller, M.

Kodikara, G.

Koga, K.T.

Kogure, $\mathrm{T}$.

Kolitsch, U.

Komabayashi, T.

Komatsu, K.

Komatsu, T.

Konrad-Schmolke, M.

Koulialias, D.

Kovacs, I.

Krivovichev, S.V.

Krüger, B.

Krüger, $\mathrm{H}$.

Kubicki, J.

Kulnitskiy, B.

Kung, J.

Kunz, B.

Kunz, M.

Kusano, Y.

Lackey, J.S.

Lai, X.

Lamb, W.M.

Lanari, P.

Lane, M.D.

Lanson, B.

Lanzirotti, A.

Layton-Matthews, D.

Le Losq, C.J.

Lee, C.-T.A.

Legros, $\mathrm{H}$.

Lenting, C.

Lesher, C.M.

Levitan, D.

Li, B.

Li, J.

Li, Y.

Libowitzky, E.

Liebske, C.

Lindsley, D.H.

Ling, $M$.

Liou, J.G.

Litasov, K.D.

Liu, F.

Liu, J.

Liu, P.

Llovet, X.

Lo Presti, L.

Lokhat, D.

London, D.

Louvel, M.

Lowenstern, J.B.

Lu, Z.

Lueth, V.

Lykova, I.

$\mathrm{Ma}, \mathrm{C}$.

Macdonald, R.

MacLennan, J.
Madden, A.S.

Majka, J.

Majumdar, A.

Majzlan, J.

Malcherek, T.

Maneta, V.

Manthilake, G.

Mao, Z.

Marcus, M.A.

Marschall, $\mathrm{H}$.

Marsh, E.

Marshall, D.D.

Martin, E.

Martin, R.

Masci, L.

Mathur, R.

Matzen, A.

Mauro, D.

Mavrogenes, J.A.

McCarthy, A.

McClenaghan, S.H.

McCollom, T.

McCoy, T.J.

McDonald, A.M.

McDonald, I.

McFarlane, C.R.

McGuire, C.

McKenna, K.

McKeown, D.A.

McMillan, P.F.

McSween, H.Y.

Mellini, M.

Menegon, L.

Mercurio, M.

Merlini, M.

Mikhail, S.

Mikouchi, T.

Miller, C.F.

Mitchell, R.

Mittlefehldt, D.

Moecher, D.

Mohammadi, N.

Molina, J.F.

Moore, D.

Moore, G.

Morard, G.

Morgan, D.

Morrison, S.M.

Moscati, R.

Mosenfelder, J.L.

Moulas, E.

Mueller, A.B.

Mungall, J.E.

Musiiachenko, K.

Mustoe, G.E

Nasdala, L.

Nassif, N.

Neave, D.A. 
Németh, $\mathrm{P}$.

Nespolo, M.

Nestola, F.

Nex, P.

$\mathrm{Ni}, \mathrm{H}$.

Ni, P.

Nicholls, J.

Nicoli, G.

Nielsen, T.

Nimis, P.

Nishihara, Y.

Nozaka, T.

O'Bannon, E.F.

Oganov, A.

Ohta, K.

Olds, T.

Olivo, G.

Osborne, Z.

Padrón-Navarta, J.A.

Pagé, L.

Paineau, E.N.

Pamato, M.

Pamukcu, A.

Panero, W.

Pannunzio Miner, E.

Paonita, A.

Parente, M.

Pasero, M.

Pasteris, J.D.

Patel, K.B.

Pekov, I.V.

Pérez-Soba, C.

Peslier, A.

Petaev, M.

Peterman, E.M.

Piccoli, P.M.

Pigott, J.

Pina, C.M.

Pingitore, N.

Pinti, D.

Pisch, A.

Plasil, J.

Plotinskaya, O.

Plotinskaya, O.Y.

Poerschke, D.

Popa, R.

Poulin, R.

Prêt, D.

Prencipe, M.

Prissel, K.B.

Puri, S.

Putnis, A.

Rakovan, J.F.

Rampe, E.

Ran, Y.
Redkin, A.

Reichmann, H.J.

Remusat, L.

Rickard, D.

Ridolfi, F.

Robinson, P.

Roddatis, V.

Rohrbach, A.

Roman, A.

Rondeau, B.

Rosiere, C.A.

Ross, N.L.

Rouwet, D.

Rowe, M.C.

Rubatto, D.

Russell, J.K.

Ruzicka, A.

Sakharov, B.A.

Sakoda, A.

Sanchez-Roa, C.

Sanematsu, K.

Sanfilippo, A.

Sanloup, C.

Sano-Furukawa, A.

Scaillet, B.

Schade, U.

Schaefer, L.

Schaefer, T.

Schindler, M.

Schlegel, M.L.

Schoenleber, A.

Schoonheydt, R.

Schranz, W.

Scott, H.P.

Scott, R.

Seitz, S.

Self, S.

Sergio Speziale, S.

Shatskiy, A.F.

Shatsky, A.

Shaw, C.S.

Shen, A.H.

Shen, Y.

Shibazaki, Y.

Shim, S.-H.

Shimizu, K.

Shinozaki, A.

Shiryaev, A.A.

Shivaramaiah, R.

Shorttle, O.

Shumilova, T.G.

Simon, A.C.

Sinmyo, R.

Sirbescu, M.-L.C.

Skála, R.
Skogby, H.

Skuzovatov, S.Y.

Sleep, N.

Smith, M.

Smye, A.

Smythe, D.J.

Sobel, E.

Song, S.

Sorensen, B.

Sossi, P.

Spear, F.S.

Spera, F.J.

Stackhouse, S.

Stagno, V.

Stanley, C.

Stebbins, J.F.

Steele-MacInnis, M.

Steinle-Neumann, G.

Stekiel, M.

Stepanov, A.S.

Stowell, H.H.

Sutton, S.R.

Tacker, C.

Tang, Y.

Tappe, S.

Teng, $\mathrm{H}$.

Terasaki, $\mathrm{H}$.

Theng, B.

Thomas, J.

Thomas, R.

Thompson, E.C.

Thompson, J.

Tollan, P.

Tomascak, P.

Tomioka, N.

Townsend, J.

Treiman, A.

Tschauner, O.

Tsujimori, T.

Tsuno, K.

Turnbull, R.E.

Umemoto, K.

Van Driessche, A.

Van Orman, J.

Van Westrenen, W.

Veksler, I.V.

Vennari, C.

Vocadlo, L.

Vukmanovic, Z.

Vural, A.

Wade, J.

Wagner, J.

Wakabayashi, J.

Walker, D.

Wall, F.
Wallace, A.

Wallace, M.W.

Walowski, K.

Wang, A.

Wang, R.C.

Wang, W.

Watkins, J.

Watson, B.B.

Watson, $\mathrm{H}$.

Webb, S.

Webb, S.M.

Wei, C.

Wei, C.

Weinberg, R.F.

Weisenberger, T.B.

Welch, M.D.

Weller, O.

Wells, M.

Wells, S.

Wenz, M.

Werts, K.

Wilkinson, J.

Wilson, K.

Winkler, B.

Withers, A.

Wolfe, O.

Wolthers, M.

Woodland, A.B.

Wright, K.

Wu, X.

$\mathrm{Wu}, \mathrm{Z}$.

Wunder, B.

Xia, Q.

Xie, G.

Xie, $\mathrm{S}$.

Xiong, F.

$\mathrm{Xu}, \mathrm{J}$.

Yakymchuk, C.

Yang, $\mathrm{H}$.

Yang, J.

Yoder, C.H.

Yoshino, $\mathrm{T}$.

Yu, C.

Yuanming, P.

Zeng, L.

Zhai, D.

Zhang, D.

Zhang, J.S.

Zhang, P.-F.

Zhang, R.

Zhang, Y.

Zhao, J.

Zhou, L.

Zhu, M. 